\title{
Non-Poisson variations in photomultipliers and implications for luminescence dating
}

\author{
J. Carter ${ }^{1,2, *}$, A.J. Cresswell ${ }^{1}$, T.C. Kinnaird ${ }^{1}$ L.A. Carmichael ${ }^{1}$, S. Murphy ${ }^{1}$, D.C.W. \\ Sanderson ${ }^{1}$ \\ ${ }^{1}$ Scottish Universities Environmental Research Centre, Rankine Av, East Kilbride, G75 0QF, UK \\ ${ }^{2}$ SUPA School of Physics \& Astronomy, Kelvin Building, University of Glasgow, Glasgow G12 8QQ, UK \\ * Corresponding author: email j.carter.1@ research.gla.ac.uk
}

\begin{abstract}
Previous studies have suggested that excess variations from single-photon counting systems used in luminescence dating may result in underestimation of errors and profoundly influence age models. In this study ten different photon counting systems have been investigated to explore this effect with a greater number of photomultiplier types and instrumental architectures. It is shown that radiation induced phosphorescence from F1 feldspar produces a controllable low-level light source whose local variance approximates Poisson expectations.
\end{abstract} However excess variation in dark counts was observed to varying extents from all systems. The excess variance is slightly anti-correlated with the age of the system, with older devices conforming more closely to Poisson behaviour. This observation does not seem to fit the hypothesis that enhanced levels of helium diffused into older tubes increase non-Poisson components. It was noted that a significant part of the non-Poisson behaviour was associated with multi-event pulse streams within time series. Work was also undertaken to develop mitigation methods for data analysis and to examine the implications for dating uncertainties in a test case. A Poisson-filtering algorithm was developed to identify and remove improbable multi-event streams. Application to data from signal-limited single grains of sediments from a Neolithic chambered tomb in Corsica has shown that, for this case, removing non-Poisson components improves the robustness of retained data, but has less 
30 influence on overall dating precision or accuracy. In signal limited applications use of this

31 algorithm to remove one source of excess variation is beneficial. The algorithm and test data

32 are appended to facilitate this.

33

34 Keywords: Single photon counting; Poisson variations; Phosphorescence; OSL single grain 35 dating; Poisson filtering

36

37 Highlights

38 - New investigation of behaviour of 10 diverse photon counting systems

- Low level phosphorescence close to Poisson counting statistics

- Dark counts show non-Poisson counting statistics

- New Poisson filter algorithm applied to low-sensitivity single grain data

- Non-Poisson dark counts affect rejection criteria but not overall dating errors 


\section{Introduction}

47 Luminescence methods measure the number of photons emitted from a sample under stimulation, and use single photon counting photo-multiplier tubes (PMTs). The

measurement backgrounds include an intrinsic detector background from the PMT in the absence of any light sources, the dark count. Generally low dark count PMTs are selected for use in luminescence instruments. A recent study (Adamiec et al., 2012) characterised the behaviour of luminescence dating systems using the EMI QA 9235 photomultiplier under low light level conditions. Three of the four systems studied showed variations in dark count in excess of the expected Poisson distribution for non-correlated random events. It was suggested that this could result in an underestimation of measurement uncertainties and have implications for age models.

The study reported here was devised to characterise a larger number and range of PMT types and instrumental architectures to produce a significant body of new data that could help provide a broader understanding of the behaviour. The implications for the accuracy and uncertainty of luminescence measurements were also studied using a case study where low levels of luminescence signal reached critical levels, and using the system within the Scottish Universities Environmental Research Centre (SUERC) dating lab which showed the greatest level of excess dark count variation. This involved single grain Optically Stimulated Luminescence (OSL) analysis of sediments from the constructional layers associated with a Neolithic chambered tomb in Corsica. The technical part of the study included development of an algorithm to identify non-Poisson behaviour associated with multi-event bursts in luminescence measurements and remove their associated artefacts by interpolation. The use of this algorithm on a set of low intensity single grain luminescence measurements allows an 
assessment of the impact of non-Poisson dark counts on measurement accuracy and precision.

Dark counts are 'false' photon counts induced by various mechanisms including thermal emission of electrons from the photo cathode and first dynode, cosmic ray interactions, ambient radiation in the environment and potentially electrical interference. Signal counts from single photo-electron emission at the photocathode coupled with electron multiplication and pulse height selection form the basis of photon counting. If these are uncorrelated random events they should follow a Poisson distribution described by the equation, $P(\lambda)=$ $e^{-\bar{n}} \frac{\bar{n}^{\lambda}}{\lambda !}$, where $\mathrm{P}(\lambda)$ is the probability of observing $\lambda$ events in a given interval, $\bar{n}$ is the average number of events per interval and $\lambda$ is the number of events (Poisson 1837, Stigler et al., 1982). A behavioural characteristic of the Poisson distribution is that the standard deviation of the distribution, $\sigma$, is equal to the square root of the number of observations $\sqrt{n}$ within a given time interval. For this study $\mathrm{n}$ is the number of photon counts per detection channel, as is the case in routine luminescence measurements. Deviations from a Poisson distribution would indicate correlated or anticorrelated components in the counting data. .

Recent work studying the behaviour of a small number of dating instruments (Adamiec et al., 2012) under dark conditions and varying light levels has shown that under illumination the observed behaviour followed Poisson statistics, however excess variance was seen in some devices under dark conditions. This work introduced a parameter denoted $\mathrm{k}$, which was the ratio of the observed standard deviation to the expected standard deviation based on poisson statistics. Three Risø readers using the EMI QA 9235 PMT showed dark-count k values in excess of unity, indicating non-Poisson behaviour, with an older Daybreak system using the same PMT showing a dark-count k value near unity, once the prescaling factor was 
considered. It is unclear from this study whether the observed non-Poisson behaviour is common in different PMT types and ages, nor what causes this behaviour. Further work elaborating the methods of estimating the equivalent dose and their uncertainties when nonPoisson variances are present is given by Bluszcz et al., 2015.

It has been suggested that afterpulses generated by electron interactions with gases inside the photomultiplier may explain the observed excess variance. Such after pulses are described in detail by Morton et al., 1967, showing that in 8575 photomultipliers under low light conditions approximately $5 \%$ of pulses have an associated afterpulse $\sim 0.3 \mu$ s after the main pulse. This was further explored by Coates (1973) using 8850 and 8852 photomultiplers, noting that the afterpulse time distribution enables ions of different masses to be separated (with the PMT acting like a crude time of flight (TOF) mass spectrometer) hence allowing the physical nature of afterpulses to be determined. Coates confirmed that the principle features of the TOF spectrum of the afterpulses was consistent with helium in the photomultipliers. Finite-difference Monte Carlo modelling of afterpulses in the 9235QA tube with a partial pressure of helium matching atmosphere, by Tudyka et al 2016, produced results concordant with the observations of an old 9235QA tube, where it was assumed helium diffusion had had sufficient time to equilibrate with atmosphere (Adamiec et al., 2012), and suggested that helium diffusion into PMTs may be a factor in the excess variance observed with such systems.

To address the issues raised by the earlier studies, further work into non-Poisson variation for a series of photon detection systems varying in age, tube type and electronic configuration, used for luminescence dating and the detection of irradiated food, has been conducted. In the previous work (Adamiec et al 2012) the oldest device appeared to conform more closely to 
120 Poisson behaviour. The varying ages in this investigation could explore whether there is a correlation with system age, in particular to explore the helium diffusion hypothesis since it is

122 expected that helium concentrations, and hence afterpulse frequency, within the tubes should 123 increase with age. To facilitate comparison with previous work, the approach of Adamiec et 124 al., 2012 to characterise non-Poisson variations using the " $k$ " value is adopted here, although 125 it is recognised that other parameters may also be beneficial. This larger study also provides a substantial data set that may be used to investigate the causes of the non-Poisson behaviour,

127 the extent to which this behaviour may influence the accuracy and precision of luminescence 128 measurements, and approaches to mitigate these effects. This has included the use of 129 phosphorescence as a low level light source, and the development of an algorithm which may 130 be used to identify non-Poisson behaviour in measurements and adjust the data to remove these effects.

\section{Investigation}

The environmental physics group at SUERC have a large number of photon counting systems that utilise different photomultipliers and architectures in different instruments. To characterise the devices, measurements were conducted with luminescence and under dark conditions. In this study, ten of these systems used for luminescence dating and the detection of irradiated foods (Sanderson et al., 1989) have been investigated, developed between 1986 and 2015. These systems are:

- Two manual TL readers developed in 1986 and 1989 (Sanderson et al., 1989, Spencer et al., 1994), designated SUERC TL readers TL1 and TL3. These use selected low dark count photomultipliers with 2" bi-alkali photocathodes and fourteen stage linear 
focused dynodes (type D295QA for TL Reader 1 and 9883QB for SUERC TL Reader $3)$.

- Two systems developed for PSL screening of food, here designated PSL 1 and PSL2. PSL1 (SURRC PPSL system serial number 8) uses a 9829B PMT with a 2" bi-alkali cathode, selected for low background rate, and uses the SURRC PPSL 1 board with a pre-amplifier/discriminator integral ETL device (Sanderson et al., 1989). PSL2 (SUERC PPSL system serial number 93) has a 9814B also a 2" bi-alkali cathode, again selected for low background rate, and uses the SUERC PPSL 2 control board with a PIC 18 microcontroller USB2 communication to Windows (Sanderson et al., 2003).

- Two OSL Portable readers using photo detector modules incorporating selected 9124B tubes with 1" photocathodes and built in HV and amplifier- discriminator systems. Both use the SUERC PPSL 2 board (Sanderson \& Murphy., 2010) for synchronised luminescence stimulation and photon counting.

- Two OSL scanning imaging instruments (OSL1 and OSL PICS) built for the detection of irradiated foods (Sanderson et al., 2001) and with selected 9883QB (OSL1) and 9883B (OSL PICS) PMTs and EMI C604 amplifier discriminators connected via ECL-TTL converters to the SURRC PPSL 1A photon counting board with 24-bit 100MHz bandwidth photon counters.

- Two Risø readers, using the 9235QA (Risø 1) and 9235QB (Risø 3) PMTs (the modern linear focussed version of the old 9635 scintillation counting venetian blind dynode EMI Tube originally used in Oxford for photon counting) with proprietary HV amplifier discriminator electronics with very small amplitude pulse to preserve amplitude (Bøtter-Jensen et al., 2000, Bøtter-Jensen et al., 2010). 


\subsection{Phosphorescence as a low level light source}

171

172 The first issue was to find a suitable random light source. Low level beta lights (such as ${ }^{14} \mathrm{C}$ )

173 may not be random due to the presence of correlated photons. The study by Adamiec etal.

174 (2012) used light emitting diodes (LEDs), however these may also be affected by non-

175 random variables related to maintaining the LED at a steady state for a prolonged period of

176 time. Other studies have used incandescent light sources with pin hole apertures to limit

177 photon emission rates into the experimental system, which may be affected by similar

178 variables as LEDs. In this work the use of phosphorescence as a low level random light

179 source was investigated. The potential advantages are that this is a light source which can be

180 readily achieved within luminescence instrumentation without incorporating additional systems, and in thermoluminescence (TL) instruments control of the temperature can be used to adjust the phosphorescence decay rate. In addition, the predictable decay of the light source allows evaluation of PMT performance under different light conditions within a single

184 experiment. Phosphorescence was achieved by irradiating an International Atomic Energy

185 Agency (IAEA) F1 feldspar sample with a ${ }^{90} \mathrm{Sr}$ beta source, and initial investigations confirmed that once irradiated and stored the phosphorescence tail could be used as a slowly decaying source controllable to produce approximately 100-200 counts per second.

189 As a decaying source, phosphorescence results in changing light levels with varying mean 190 count rates coupled with random counting variations. However by fitting decay curves and examining residuals in conjunction with local decay rates the random variation components as a function of light level can be estimated. In this work phosphorescence decays were fitted by single exponentials and residuals calculated by subtracting the calculated from the

194 measured counts, as illustrated in Fig.1. The standard deviation of the residuals was taken as 
the observed error of the system, at the corresponding intensity of the signal. The approach is similar to that taken by Adamiec et.al. 2012, where a second order polynomial function was used to de-trend light source measurements, with the variance on the residuals used as the measured variance. To assess the extent to which this approximation to the statistical behaviour of varying light sources can be relied on, a single phosphorescence measurement (from the SUERC Portable OSL Reader) was divided into shorter time intervals, each corresponding to a $1 \%$ decay in phosphorescence as determined from the fitted exponential function, with the k-value calculated for each interval using the standard deviation on the measured photon counts rather than the residuals. The k-values calculated for each data segment are plotted in Fig 2, with the k-value calculated from the residuals for the entire measurement for reference. It can be seen that the segmented values scatter around the value for the entire measurement, with high values corresponding to significant excess counts at approximately 100 and 450s. The mean of the k-values for all segments $(1.111 \pm 0.002)$ compares favourably for the k-value from the entire measurement $(1.117 \pm 0.002)$. Rejecting the segments with excess counts brings the value of $\mathrm{k}$ closer to unity $(1.03 \pm 0.001)$.

The data in Fig. 1 has been selected as having a relatively rapid signal decay, approximately $25 \%$ over the measurement (from $\sim 100$ to $\sim 75 \mathrm{cps}$ ), so that the curve fitting could be observed. Other measurements showed much slower decays, approximately $10 \%$ over 600 s. Thus, it can be seen that it is possible to generate low-level phosphorescence light sources which decay slowly, in order to study residual variations under controlled conditions from simple and highly predictable sources of low level light. 


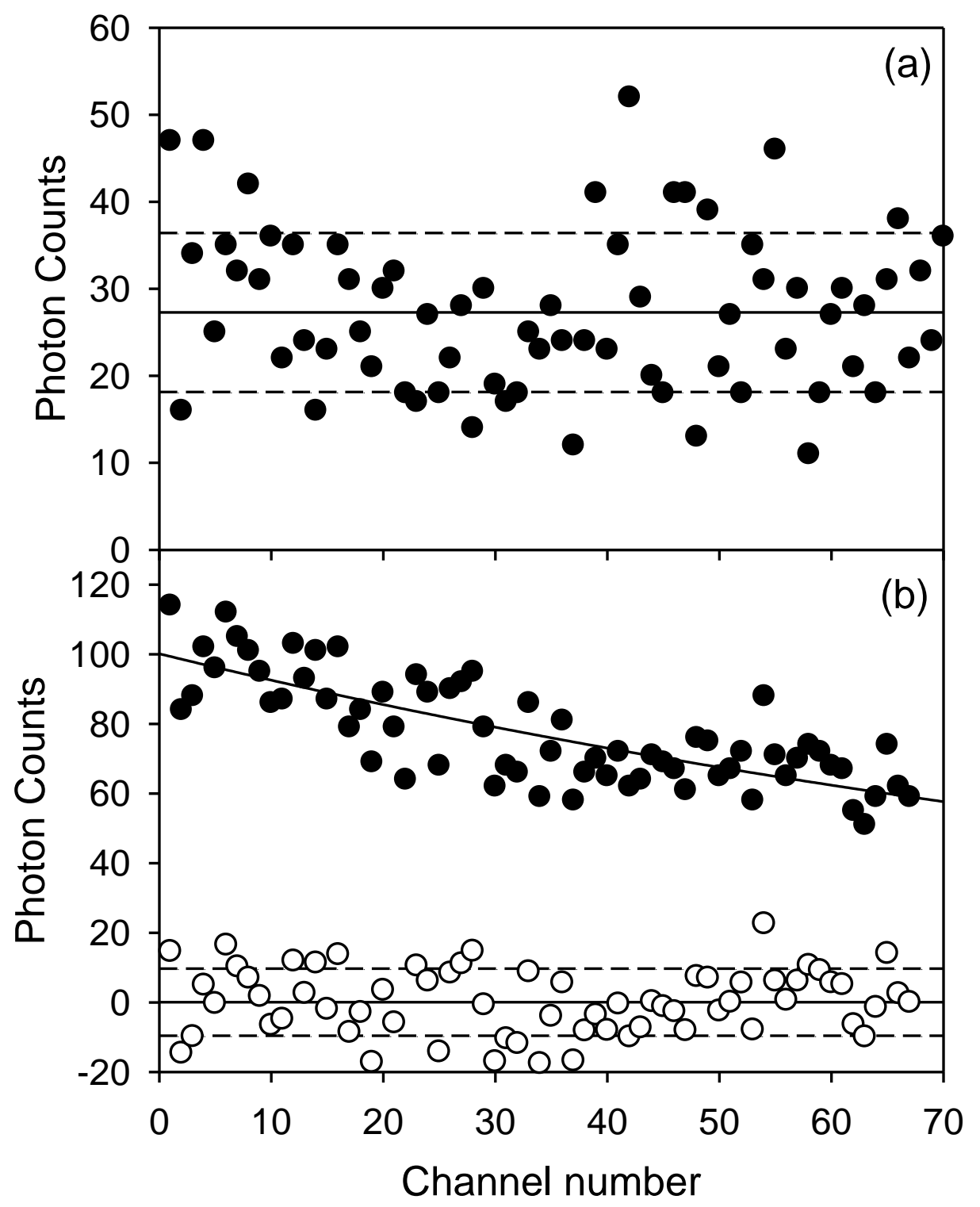

219 Fig 1. Example selected interval spectra for the SUERC Manual TL Reader. a) dark count,

220 showing mean and standard deviation, and b) phosphorescence, showing measured data

221 (filled circles) and fitted exponential decay and residuals (open circles) with mean and 222 standard deviation. 

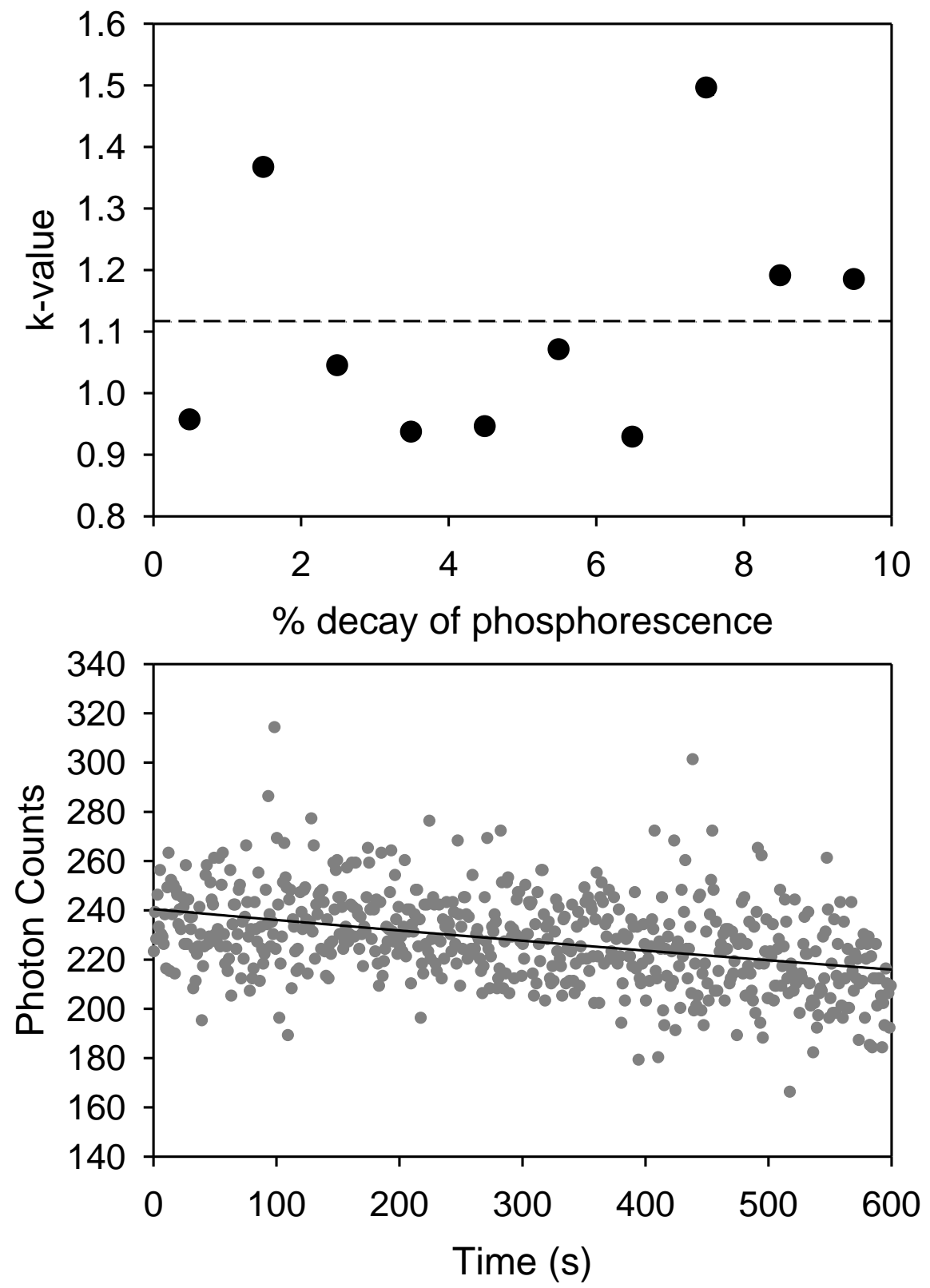

224 Fig. 2. k-values calculated for segments corresponding to $1 \%$ of the phosphorescence decay

225 measured on OSL Portable 1, with the k-value for the entire measurement indicated by the 226 dashed line. Larger k-values correspond to points in the measurement with higher counts 227 (lower plot). 


\subsection{Investigations of PMT response}

232 For this investigation multiple measurements of 600 seconds were performed under

233 phosphorescence and dark conditions for each device. Examples of low light level with the

234 fitted exponential decay and dark count spectra are shown in Fig. 1. Following the

235 characterisation process of Adamiec et al., 2012, each device was characterised a k-value

236 defined as $k=\sigma / \sqrt{\bar{N}}$, the ratio of the observed standard deviation ( $\sigma$ ) from a series of

237 measurements and the standard deviation that is expected from Poisson statistics $(\sqrt{\bar{N}})$.

238 Adamiec et al., 2012 plot k-values with uncertainties, but the paper does not state how these uncertainties were estimated. Here we estimate these uncertainties by taking the uncertainty on $\bar{N}$ to be the standard error $(\sigma / \sqrt{n})$ where $\mathrm{n}$ is the number of measurements in the data set, and approximate the fractional uncertainty on $k$ to half the fractional uncertainty on $\bar{N}$. Thus

$$
\Delta k \approx k(0.5(\sigma / \sqrt{n}) / \bar{N})
$$

243 It is noted that the uncertainties on $k$ thus estimated are similar to those for k-values plotted by Adamiec et al 2012 .

246 For an ideal detection system and random light source Poisson statistics are expected leading

247 on average to k-values of 1 .Values significantly different than one indicate non-random

248 variations, with values greater than one corresponding to excess variation..

Dark counts were measured on all devices, by running the closed systems without light sources, and k-values calculated using the standard deviation and the mean counts for the measurements. Then phosphorescence measurements were conducted at low levels and k-

253 values calculated using the standard deviation on the residuals and the mean counts for the measurements. 


\section{Results of PMT response investigations}

256

257 Full k parameter results under phosphorescence and dark conditions for all devices are shown

258 in Table 1 with their corresponding development ages, tube types and mean dark count rates.

259

260 Under low light conditions all devices have k values close to unity, and thus show the expected Poisson behaviour. Under dark conditions, k values with the possible exception of

262 the oldest unit, are greater than unity indicating excess variance compared with Poisson

263 statistics for all photomultipliers to varying extents. These results corroborate and extend the

264 findings of Adamiec etal 2012 and indicating that similar phenomena can be observed across

265 a wider range of PMT types and architectures. 


\begin{tabular}{|c|c|c|c|c|c|c|c|}
\hline \multirow[t]{2}{*}{ Photomultiplier } & \multirow{2}{*}{$\begin{array}{c}\text { System } \\
\text { Development } \\
\text { Age }\end{array}$} & \multirow[t]{2}{*}{ PM Type } & \multicolumn{3}{|c|}{ Dark count measurements } & \multicolumn{2}{|c|}{ Phosphorescence measurements } \\
\hline & & & $\begin{array}{c}\text { Number of } \\
\text { Measurements } \\
(600 s)\end{array}$ & $\begin{array}{c}\text { Mean } \\
\text { Count Rate } \\
(\mathrm{cps}) \pm \text { std dev }\end{array}$ & $k_{D C} \pm \sigma$ & $\begin{array}{c}\text { Number of } \\
\text { Measurements } \\
\text { (600s) }\end{array}$ & $k_{p} \pm \sigma$ \\
\hline SUERC Manual TL 1 & 1986 & D295QA & 7 & $53 \pm 10$ & $1.37 \pm 0.01$ & 3 & $0.89 \pm 0.01$ \\
\hline Reader TL 3 & 1989 & 9883QB & 5 & $28 \pm 9$ & $1.70 \pm 0.01$ & 3 & $1.00 \pm 0.01$ \\
\hline PSL 1 & 1989 & 9829B & 5 & $17 \pm 7$ & $1.70 \pm 0.01$ & 1 & $1.07 \pm 0.06$ \\
\hline PSL 2 & 1989 & 9814B & 5 & $25 \pm 11$ & $2.20 \pm 0.02$ & 1 & $0.97 \pm 0.03$ \\
\hline OSL & 2001 & 9883QB & 6 & $27 \pm 10$ & $1.92 \pm 0.02$ & 2 & $0.92 \pm 0.09$ \\
\hline OSL PICS & 2001 & 9883B & 7 & $23 \pm 10$ & $2.08 \pm 0.01$ & 2 & $0.91 \pm 0.08$ \\
\hline OSL Portable 1 & 2010 & 9124B & 8 & $8 \pm 8$ & $2.82 \pm 0.06$ & 2 & $0.82 \pm 0.03$ \\
\hline OSL Portable 2 & 2015 & $9124 \mathrm{~B}$ & 8 & $10 \pm 4$ & $1.26 \pm 0.01$ & 2 & $0.89 \pm 0.05$ \\
\hline Ris $\varnothing 1$ & 1999 & 9235QA & 7 & $65 \pm 18$ & $2.23 \pm 0.01$ & 1 & $1.16 \pm 0.07$ \\
\hline Risø 3 & 2008 & 9235QB & 10 & $49 \pm 23$ & $3.28 \pm 0.03$ & 1 & $1.01 \pm 0.06$ \\
\hline
\end{tabular}

266 Table 1.

267 Summary of results for all PMT's, showing mean dark count rates, and k-values for the phosphorescence and dark count measurements. 
268 Fig 3 plots the $k_{D C}$ parameter against the year each device was produced and suggests a slight

269

270

271

272

273

274

275

276

277

278

279

280

281

282

283

284

285

286 correlation (younger devices showing a greater extent of non-Poisson behaviour). It has been suggested that afterpulse rates associated with helium which had diffused into the PMT may be responsible for the non-Poisson behaviour. In this case it might be expected that under comparable diffusion rates the oldest systems should contain more helium than the younger ones. Since the afterpulse intensity is a function of helium concentration then older systems should be more susceptible to non-Poisson behaviour. The data do not support this hypothesis. Nor is there a simple relationship between the use of quartz windows or glass windows, which are expected to show different helium diffusion rates and the excess variance observed. Both these observations suggest that other factors than helium concentration are likely to be involved in the excess dark count variations observed.

Fig 4 presents a comparison between spectra recorded under dark conditions for the device most closely following Poisson statistics (SUERC manual TL Reader) and the least well behaved device (Risø 3). The Risø 3 spectrum shows high single channel bursts, with correspondingly long tail to high photon counts, above 100, in the histogram. The SUERC manual TL reader has a lower dark count and does not include single channel bursts of comparable amplitude. The non-Poisson component in the Ris $\varnothing 3$ system is largely associated with these single channels with photon counts significantly in excess of Poisson expectations. 


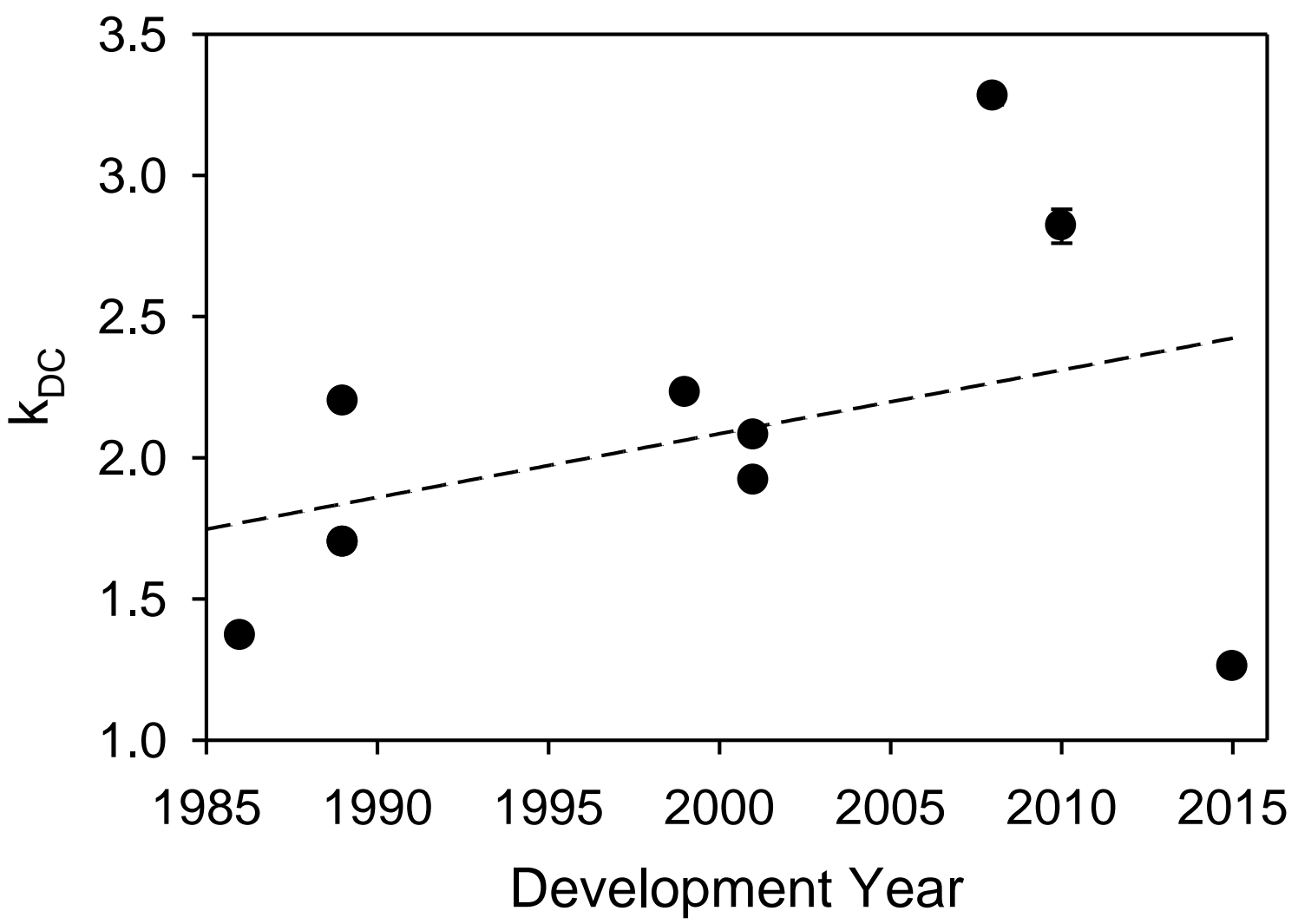

287

288 Fig 3. Age correlation graph; readers from left to right (SUERC Manual TL Reader, TL

289 Reader 2, PSL 1, PSL 2, OSL, OSL PICS, OSL Portable 1, OSL Portable 2, Ris $\varnothing$ 1, Ris $\varnothing$ 3)

290 measured $k_{D C}$ parameter plotted against the development year of the devices, with a

291 correlation line (dashed-line plotted).

292 

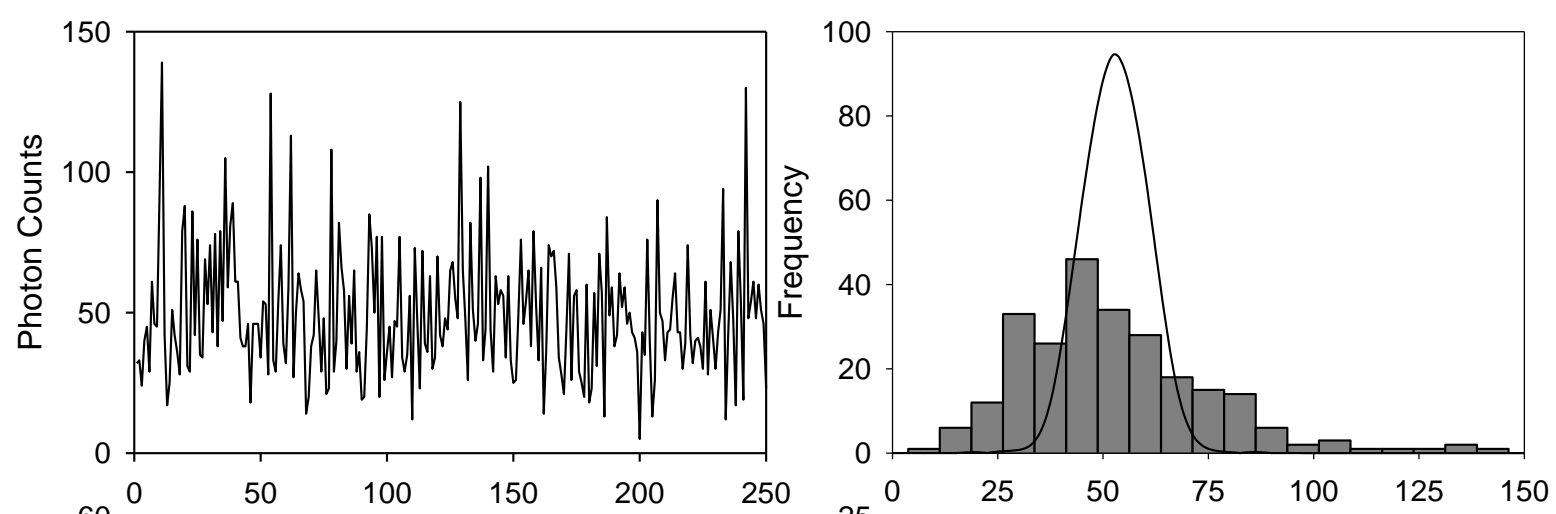

293
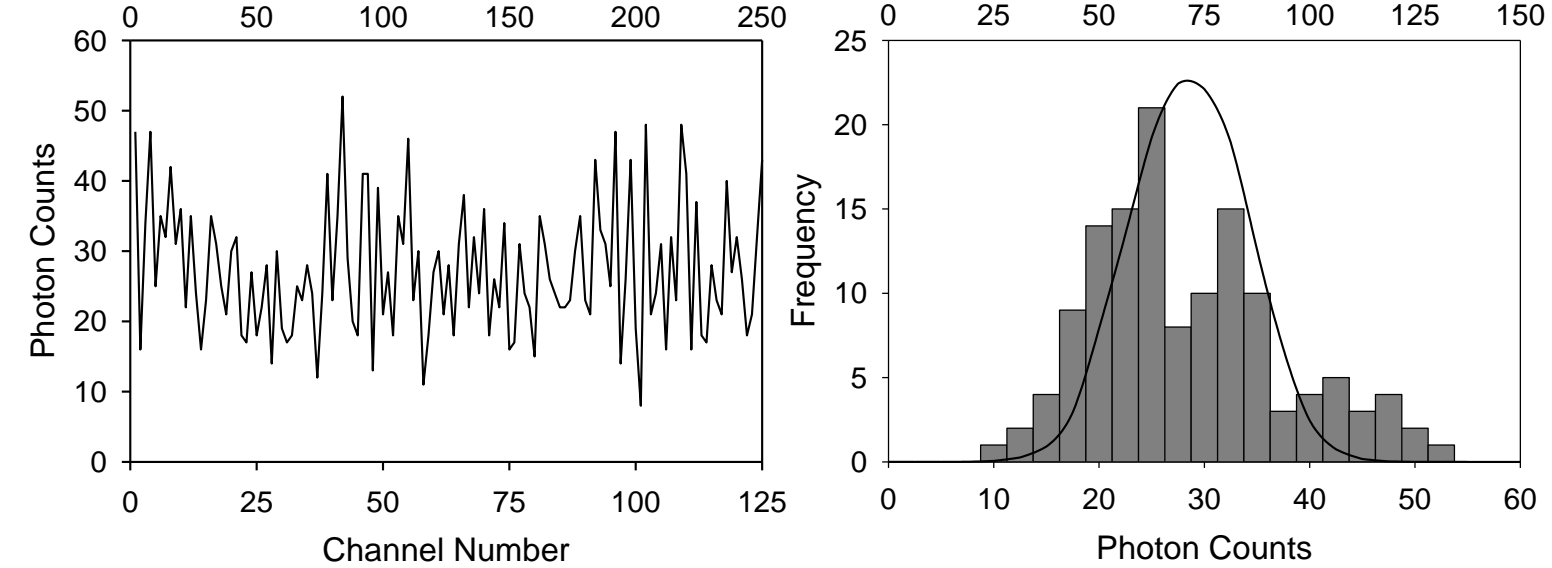

294 Fig 4. Comparison of the dark count spectra between the systems showing the most and least excess variance (Risø 3, top, and SUERC Manual TL Reader, bottom). Solid line is the fitted

Poisson probability distribution of the counts detected.

\section{Poisson Smoothing}

\subsection{Development of algorithm}

303 Although the cause of excess variance in some systems is still unclear, it is noted that the excess variances at or close to dark count largely manifest as single channel spikes with counts significantly in excess of the expectations from Poisson statistics. This leads to the possibility of an algorithm to identify and remove these spikes, and hence reduce the excess 
variation. A Poisson filter algorithm (illustrated in Fig. 5, with the script and test data in

308 Supplementary Information) has been written which calculates the probability of the counts

309 in a given channel falling within a Poisson distribution defined by the mean and standard

310 deviation of the spectrum. Any isolated channel counts with a Poisson probability below an

311 acceptable value (which can be input by the user) are averaged out with four neighbouring

312 channels, thus smoothing out the counts that are single channel bursts not following Poisson

313 statistics. Multiple adjacent channels below the acceptance criteria, which would include

314 signals from mineral grains, are not affected. In Figure 6 the application is implemented on

315 the Risø 3 dark count spectra, the counts that are out with Poisson criteria are removed and

316 averaged. In this case this has reduced the $k_{D C}$ parameter for from $3.21 \pm 0.05$ to $2.38 \pm 0.03$,

317 thus the filter has removed some of the excess variation.

318

319

320

321

322

323

324

325 


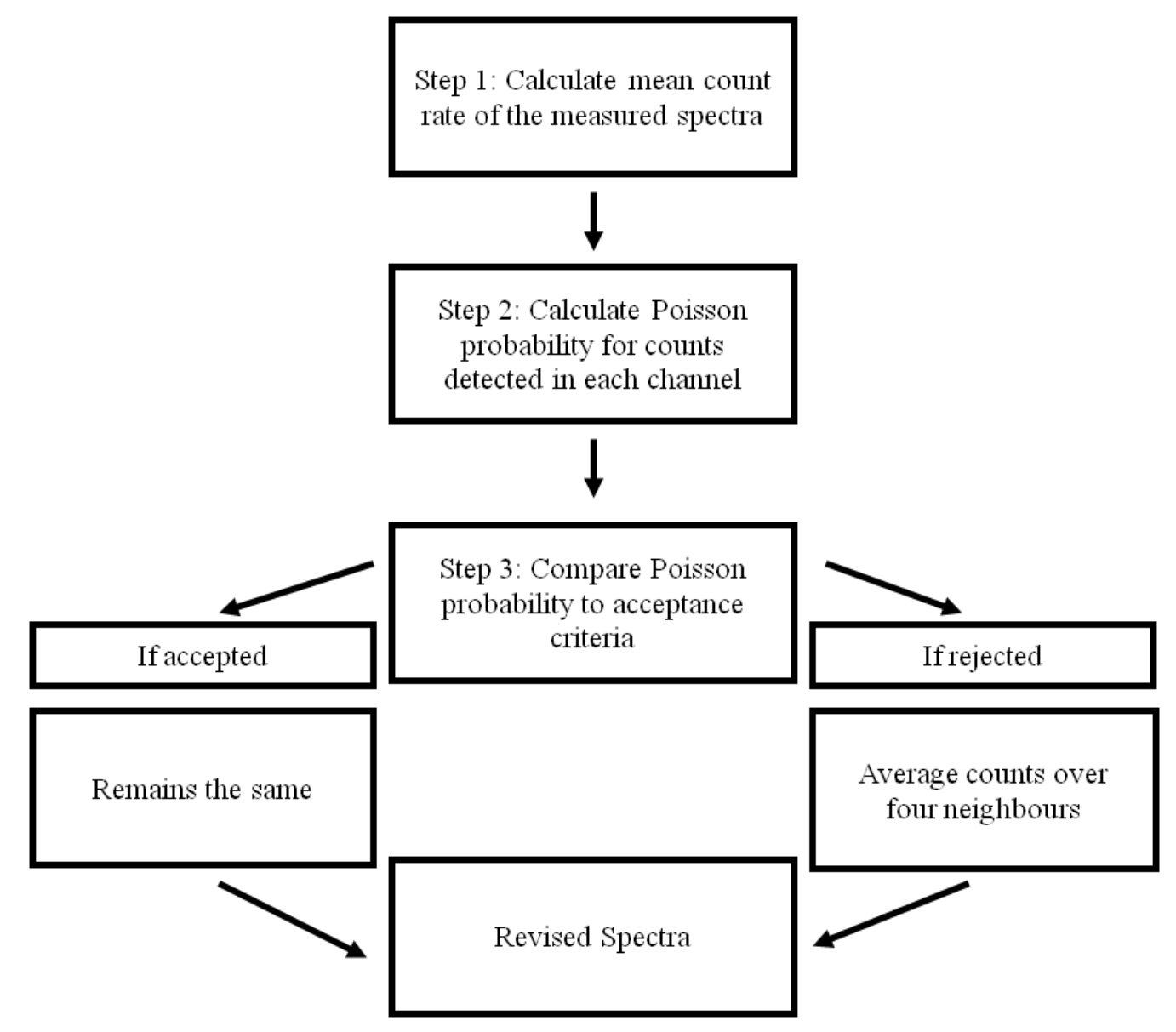

327 Fig 5. Flow chart of the Poisson smoothing algorithm 


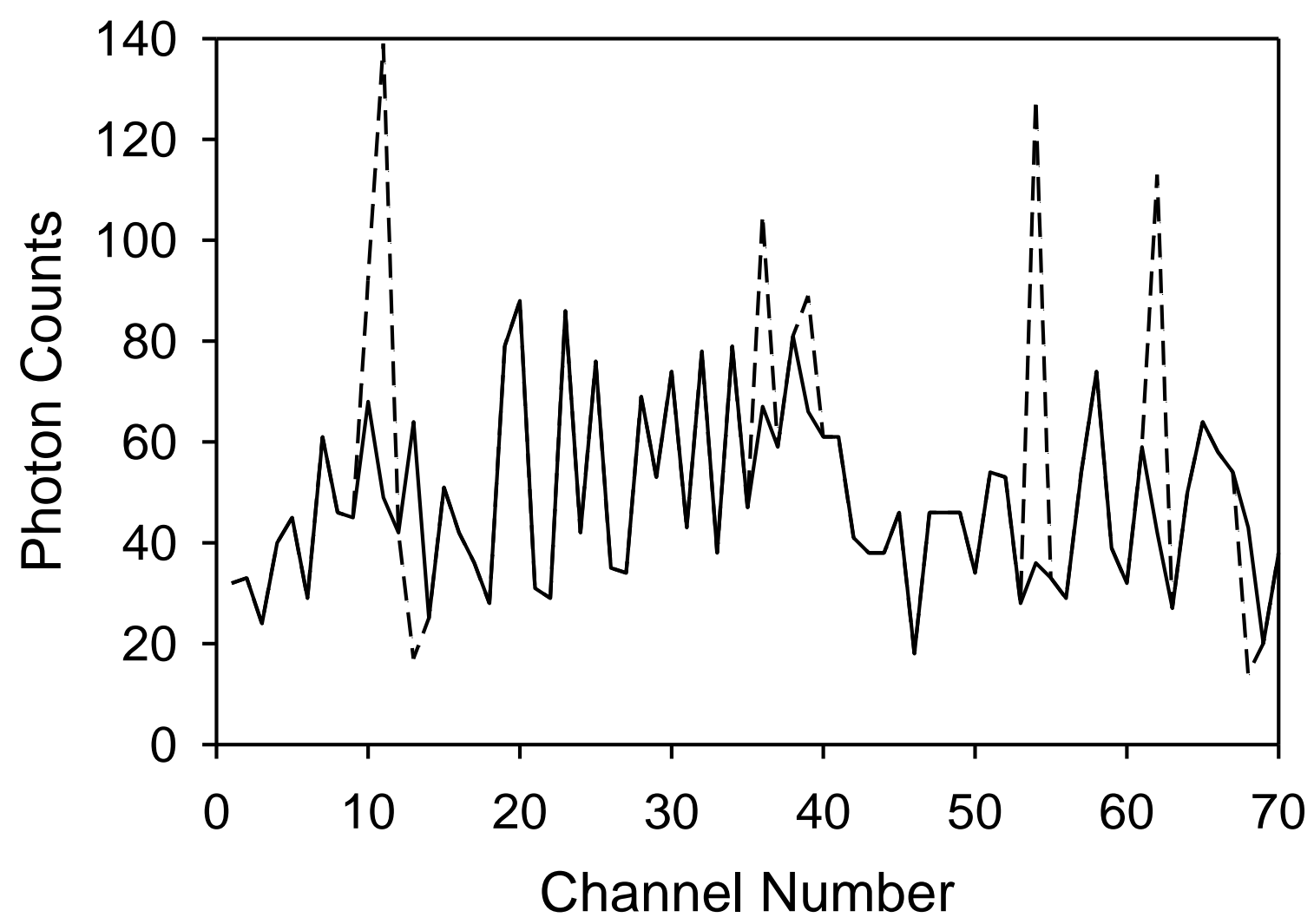

329 Fig 6. Original (dashed) and revised (solid) spectra implementing Poisson smoothing. The 330 large single channel noise bursts have been removed and averaged.

334 The Poisson filter has been applied to a test set of single grain measurements. These were

335 taken from a study of Neolithic burial chamber on Corsica (Sanderson et al., 2014, Cresswell 336 et al., 2016). The Capu di Locu project collected 92 small samples from 10 sequences, 6

337 associated with a menhir standing stone (Stantare) and 4 associated with a chambered tomb

338 (Tola).Field and laboratory profiling analysis was used to target undisturbed sedimentary

339 units with potential to date the primary construction of these Neolithic monuments. Nine

340 samples, five from Stantare and four from Tola, were collected for OSL dating. These 
341 showed low quartz OSL sensitivities of 200 - 800 integrated counts/mg/Gy for the Tola

342 samples, with higher sensitivities of 2000 - 20000 integrated counts/mg/Gy for the Stantare

343 samples. There was evidence from SAR analysis of multi-age components in the samples

344 collected from the lower fill around the Stantare menhir, and two of these samples were

345 carried forward for single grain analysis; SUTL2683 with 18 single grain disks and

346 SUTL2680L with 7 disks, in both cases using 250-500 $\mu$ m quartz. A sample from the Tola

347 site (SUTL2960B), which did not show evidence for multi-age components in the SAR

348 analysis, was used as a control sample with 7 disks used for 150-250 $\mu \mathrm{m}$ and 7 disks of 250-

$349500 \mu \mathrm{m}$ quartz grains. The single grain analyses this comprised SAR analysis of $3900 \mathrm{SG}$

350 holes each producing a series of OSL decay curves.

351

352 This case study here used measurements from the low sensitivity control sample set of seven

353 single-grain discs populated with $250-500 \mu \mathrm{m}$ quartz grains from a thin horizon below the

354 principal slab of the Tola chambered tomb (sample SUTL2960B). Measurements were

355 conducted on a Risø DA-20 automatic reader designed for single grain luminescence dating

356 (Risø 3, which has been observed to have the largest k value in this study, Table 1). Each

357 single-grain measurement consisted of four OSL measurements; the natural and a 25 Gy

358 regenerative dose, with 5 Gy test doses. Following the 5 Gy test dose, $60 \%$ of measurements

359 produced less than 10 counts, with $37 \%$ giving $10-100$ counts and only $3 \%$ giving $100-1000$

360 counts. Acceptance criteria were based on the statistical significance of the net counts from

361 the regenerative dose compared with their estimated error-in signal uncertainty. Of the 700

362 measurements, examination under an optical microscope showed approximately half were

363 from unoccupied holes in the single grain discs, and 88 carried statistically significant

364 signals, when compared with a rejection threshold (expressed as number of standard

365 deviations) based on the estimated uncertainties of their net counts after late-light subtraction. 
366 The estimated uncertainties in this process are based on Poisson expectations. This data set

367 was chosen because the minerals had relatively low sensitivities, and hence the signals

368 observed were small and a high proportion of observations fell below 2 sigma significance

369 levels and were rejected on the basis of Poisson criteria from the conventional analysis. It

370 was considered that a case of this type would be most sensitive to non-Poisson variation in

371 comparison with dating data sets with higher signal levels.

372

373 The analysis method integrates the counts in the rapidly decaying OSL peak in the early part

374 of each measurement, and subtracts the integrated counts in the background from the end of

375 each measurement to produce a net count. Non-Poisson artefacts in the early part of the

376 measurement would inflate the net count, and removing them would reduce the statistical

377 significance of the measurements. Conversely, they would reduce the net count if present in

378 the later part of the measurement and removing them would increase the measurement

379 significance.

380

381 Poisson filtering was applied to the data set of 2800 decay curves, and filtered decay curves reanalysed and compared with the original unfiltered analysis. Figure 7 illustrates the natural and regenerated signal for a pair of these 2800 decay curves. Single channel features in the original data (dashed lines) have been removed using the filtering algorithm, and the solid

385 line shows the revised smoothed data. This shows the presence of single channel spikes

386 characteristic of non-Poisson dark count bursts which the filter has removed, leaving the "corrected" OSL signal. For this particular grain, the filter has reduced the net natural signal by removing a spike at channel 15 , but left the net regenerated signal unchanged since the spike at channel 26 does not fall within either the signal or background integrals. It can be 


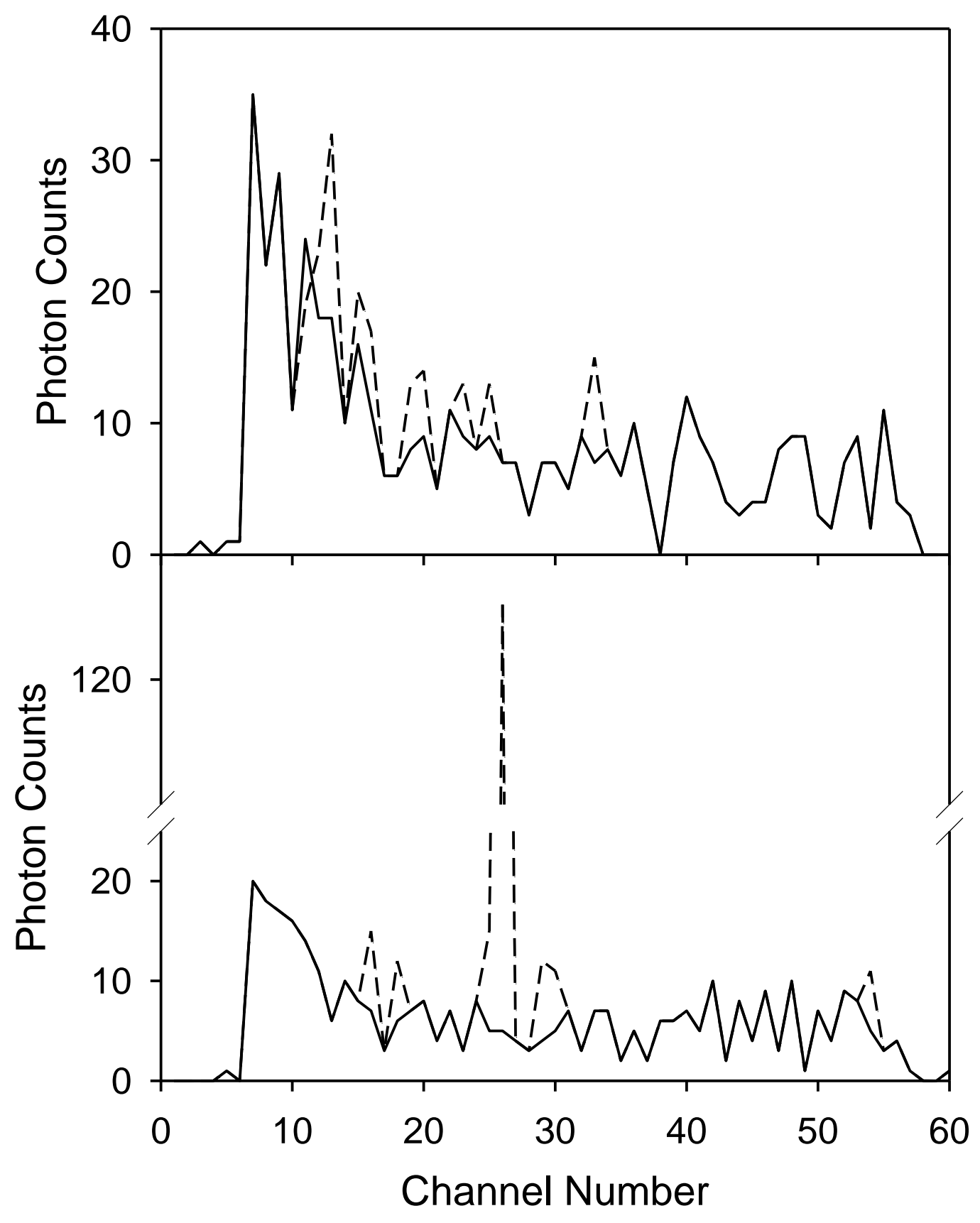

394 Fig 7. An example of one single grain signal and regenerative signal spectra, with original 395 signal (dashed line) and the Poisson smoothed signal (solid line). 
The application of the Poisson filter to this data set is summarised in Table 2, and has removed several measurements where the apparent signal is identified as a non-Poisson artefact, reducing the number of statistically significant measurements to 56. In this instance, this has not significantly changed the age calculated for this sample, although it has brought it

401 into closer agreement with the age from the original SAR analysis based on small aliquots.

402 Having removed identified artefacts from the data there will be improved confidence in the 403 equivalent dose values obtained. It is likely that other sources of over dispersion, for example micro dosimetry or partial bleaching, dominate in this instance. However, for other samples it's possible that these other sources may be less significant in which case the non-Poisson 406 behaviour of the PMT could be more important. It is therefore recommended that a filter to 407 reduce the effects of non-Poisson behaviour be routinely applied, especially to cases where 408 light levels are close to detection limits.

409

410

411 Table 2. Tola burial chamber results, showing the single grain and SAR dates for the original 412 analysis, and the single grain age following Poisson filtering.

$\begin{array}{lll}\text { Date } & \text { Number of accepted } & \text { Stored Dose } \\ & \text { grains }(250-500 \mu \mathrm{m}) & \text { Estimates }(\mathrm{Gy})\end{array}$

$\begin{array}{lrrr}\text { Original } & 2735 \pm 500 \mathrm{BC}(\mathrm{SG}) & 88 & 25.2 \pm 3.9(\mathrm{SG}) \\ & 2610 \pm 930 \mathrm{BC}(\mathrm{SAR}) & 27.0 \pm 5.3(\mathrm{SAR})\end{array}$

$\begin{array}{llll}\text { Revised } & 2670 \pm 900 \mathrm{BC} & 56 & 26.0 \pm 5.0\end{array}$ 


\section{Discussion and Conclusion}

415

416 The findings of Adamiec et al., 2012, that some photon counting systems display non-

417 Poisson dark count behaviour, have been confirmed and extended to cover a further 10

418 systems with a range in age, architecture and electronic configuration. The larger number of systems studied allows a comparison between the age of the system and the extent of nonPoisson variation. This shows a slight correlation, with younger devices showing larger excess variation.

422

423 To assess whether the electronic architecture might be influential (in particular the pulse width of the preamplifier) the PMT from our Riso3 single grain reader, which showed the greatest " $\mathrm{k}$ " value observed here was temporarily relocated to run in the electronics of our 1986 TL manual reader, which had shown the smallest " $k$ " value. The outcome of that test was that the " $k$ " value was not significantly changed by substitution to the older electronics set up. This may be taken to imply that individual PMT's have different underlying behaviour.

The use of phosphorescence obtained by irradiating a feldspar sample demonstrates a simple way of obtaining a controllable and predictable low level light source exhibiting random

433 variations around its value. The decay of this light source would allow evaluation of PMT 434 characteristics under different signal levels. The non-random phosphorescence decay can been accounted for by fitting an appropriate exponential function, with the standard deviation

436 of the residuals, coupled with the applicable light level resulting in k value estimates. This 437 approximation to a full statistical accounting of the data has been shown to have minimal 438 effects on the k-values compared to those calculated for the measurements over shorter 
439 periods where the phosphorescence decays by $1 \%$. The use of low level light sources

440 significantly reduces the calculated k-values compared with dark counts, implying that there

441 may be different processes involved in the light detection and dark signal origins in respect of

442 non-Poisson behaviour.

443

444 In our data it was evident that significant excess variation in routine observations is

445 associated with single channel event bursts. Poisson filtering of data sets from both dark

446 counts and OSL signals, showing that the filter successfully removes the single channel

447 bursts with low random probability and reduces residual non-random components.

448 Adamiec et al., 2012 and Bluscz et al 2015 had suggested that the excess variance in dark

449 counts results in underestimation of dating errors, and influences the outputs of some age

450 models. The implementation of the filter algorithm to a case study data set for a burial

451 chamber in Corsica, France, shows that in this case removal of excess variation via this

452 method had a limited effect on the uncertainties and calculated age of the sample. In this

453 case, and not-withstanding the low signal levels involved, where the dominant dating

454 uncertainties are derived from the variations in underlying dose distribution and

455 microdosimetry rather than the propagation of estimated measurement errors, the impact of

456 the non-Poisson component on error estimates and ages is not appreciable. Here the main

457 impact relates to definition of detection limits and rejection of insignificant data. Use of the

458 Poisson filter results in a more stringent rejection of low significance observations within the

459 dataset, and in our view produced a more robust analysis that obtained using the uncorrected

460 data set. The filtering algorithm and test data sets have been included to facilitate uptake for

461 those wishing to apply similar methods.

462

463 The origins of the non-random components in dark signals are not entirely clear at this stage. 
464 Our results do not fit the hypothesis that afterpulses resulting from helium diffusion are the 465 major explanatory factor in determining the extent of non-Poisson behaviour in dark signals.

466 The data sets for the older systems, which would be expected to have acquired higher helium

467 partial pressures, show less excess variation, and as noted above there is no sign that the

468 quartz windows tubes show higher $\mathrm{k}$ values than the glass systems. While afterpulses associated with helium have been observed in many systems, and typically account for a few

470 percent of signal events, the relationships between light and dark signals are less clear.

471 Tudyka et.al. 2016 have shown simulated trains of up to 8 helium linked afterpulses in small

472 proportions of events, but it is not clear whether event chains of 100 or more pulses could be

473 explained by such mechanisms, and if so what initiating and propagation events would be

474 implied. Dark response behaviour, and the ways in which dark signals change with time

475 following over-exposure of different tubes vary markedly from system to system. The role of

476 cosmic radiation, or sources of ionising radiation in proximity to the detectors in dating

477 systems may also warrant further attention. We therefore conclude that further research into

478 the behaviour of dark signals would be needed to clarify the origin and nature of non-Poisson

479 behaviour in these systems. Meanwhile Poisson filtering algorithms may be useful in helping 480 to deal with data sets close to detection limits. 


\section{References}

486

487 Adamiec, G., Heer, A.J. and Bluszcz, A., 2012. Statistics of count numbers from a

488 photomultiplier tube and its implications for error estimation. Radiat.Meas. 47(9), pp.746-

489751.

490

491 Akgun, U., Ayan, A.S., Aydin, G., Duru, F., Olson, J. and Onel, Y., 2008. Afterpulse timing 492 and rate investigation of three different Hamamatsu Photomultiplier Tubes. J. Instrum. 3(01), 493 p.T01001.

494

495 Bluszcz, A., Adamiec, G. and Heer, A.J., 2015. Estimation of equivalent dose and its 496 uncertainty in the OSL SAR protocol when count numbers do not follow a Poisson 497 distribution. Radiat.Meas, 81, pp.46-54.

498

499 Bøtter-Jensen, L., Bulur, E., Duller, G.A.T. and Murray, A.S., 2000. Advances in 500 luminescence instrument systems. Radiat.Meas, 32(5), pp.523-528.

501

502 Bøtter-Jensen, L., Thomsen, K.J. and Jain, M., 2010. Review of optically stimulated 503 luminescence (OSL) instrumental developments for retrospective 504 dosimetry. Radiat.Meas, 45(3), pp.253-257.

505

506 Clark, R.J. and Sanderson, D.C.W., 1994. Photostimulated luminescence excitation 507 spectroscopy of feldspars and micas. Radiat.Meas, 23(2-3), pp.641-646. 
509 Coates, P.B., 1973. The origins of afterpulses in photomultipliers. J. Phys. D Appl.

510 Phys. 6(10), p.1159.

511

512 Cresswell, A.J., Kinnaird, T.C., Sanderson, D.C.W., 2016. Exploratory Single Grain OSL

513 Analysis of Sediments from Capu di Locu, Corsica. SUERC Technical Report.

514 http://eprints.gla.ac.uk/159270/

515

516 Höbel, M. and Ricka, J., 1994. Dead-time and afterpulsing correction in multiphoton timing

517 with nonideal detectors. Rev.Sci.Instrum, 65(7), pp.2326-2336.

518

519 Kang, Y., Lu, H.X., Lo, Y.H., Bethune, D.S. and Risk, W.P., 2003. Dark count probability

520 and quantum efficiency of avalanche photodiodes for single-photon detection. Appl. Phys.

521 Lett, 83(14), pp.2955-2957.

522

523 Lo, C.C. and Leskovar, B., 1983. Afterpulse time spectrum measurement of RCA 8850

524 photomultiplier. IEEE T. Nucl. Sci., 30(1), pp.445-450.

525

526 Morton, G.A., Smith, H.M. and Wasserman, R., 1967. Afterpulses in photomultipliers. IEEE

527 T. Nucl. Sci., 14(1), pp.443-448.

528

529 Poisson, S.D., 1837. Probabilité des jugements en matière criminelle et en matière civile,

530 précédées des règles générales du calcul des probabilitiés. Paris, France: Bachelier, 1, 531 p.1837.

532 
533 Raikov, D., 1937. A characteristic property of the Poisson laws. In Dokl. Akad.

534 Nauk.(USSR) (Vol. 14, pp. 9-12).

535

536 Sanderson, D.C.W., Kinnaird, T.C., Cresswell, A., Leadri, F., Leandri, C., 2014. OSL dating

537 of Neolithic Monuments Capu di Locu, Belvédére, SW Corsica. SUERC Technical Report.

538 http://eprints.gla.ac.uk/110538/

539

540 Sanderson, D.C., Carmichael, L.A. and Fisk, S., 2003. Photostimulated luminescence

541 detection of irradiated herbs, spices, and seasonings: international interlaboratory trial. $J$.

542 AOAC Int. 86(5), pp.990-997.

543

544 Sanderson, D.C.W., Carmichael, L., Murphy, S., Whitely, V., Scott, E.M. and Cresswell, A.,

545 2001. Investigation of Statistical and Imaging Methods for Luminescence Detection of

546 Irradiated Ingredients. http://eprints.gla.ac.uk/58359/

547

548 Sanderson, D.C. and Murphy, S., 2010. Using simple portable OSL measurements and

549 laboratory characterisation to help understand complex and heterogeneous sediment

550 sequences for luminescence dating. Quat. Geochronol. 5(2), pp.299-305.

551

552 Sanderson, D.C.W., Slater, C. and Cairns, K.J., 1989. Thermoluminescence of foods: origins

553 and implications for detecting irradiation. Int. J. Rad. Appl. Instrum. C. 34(6), pp.915-924.

554

555 Spencer, J.Q. and Sanderson, D.C., 1994. Mapping thermal exposure by luminescence

556 thermometry. Radiat.Meas, 23(2-3), pp.465-468.

557 
558 Stigler, S.M., 1982. Poisson on the Poisson distribution. Stat. Probabil. Lett. 1(1), pp.33-35.

559

560 Tudyka, K., Adamiec, G. and Bluszcz, A., 2016. Simulation of He+ induced afterpulses in 561 PMTs. Rev.Sci.Instrum. 87(6), p.063120.

562 